\title{
The evolution of violence and aggression today: an integral analysis of the conduct of deviance
}

\section{L'evoluzione della violenza e dell'aggressività ai giorni d'oggi: analisi integrale della condotta della devianza}

\section{La evolución de la violencia y la agresión en la actualidad: un análisis integral de la conducta de desviación}

\author{
Martina Bochicchio \\ Dottoressa Magistrale in Investigazione, Criminalità e Sicurezza Internazionale, Università degli Studi Internazionali \\ di Roma, Italy
}

\begin{abstract}
For centuries philosophers, playwrights and theologians have been discussing this issue: what makes people evil? Being aggressive is an inherent aspect of man. Aggressiveness is deeply part of a person's very nature. What governs and directs it, the preconditions that incentivize and empower it, and the processes that make it chronic, continue to be subjects of investigation in the science of the mind.
\end{abstract}

\section{RIASSUNTO}

Filosofi, drammaturghi, teologi si sono confrontati per secoli su questo tema: cosa rende le persone malvagie? L'essere aggressivo è un aspetto connaturato all'uomo. L'aggressività fa profondamente parte della natura stessa di una persona. Ciò che la governa e dirige, i presupposti che la incentivano e potenziano e i processi che la rendono cronica, continuano a essere materia di indagine nel ramo delle scienze della mente.

\section{RESUMEN}

Filósofos, dramaturgos y teólogos llevan siglos debatiendo esta cuestión: ¿qué hace que las personas sean malas? Ser agresivo es un aspecto inherente al hombre. La agresividad forma parte de la propia naturaleza de una persona. Lo que la gobierna y dirige, los supuestos que la incentivan y potencian, y los procesos que la hacen crónica, siguen siendo temas de investigación en la rama de las ciencias de la mente.

Non esiste una definizione univoca del concetto di aggressività in quanto assume funzioni diverse a seconda che questa venga analizzata da un'ottica di tipo psicologico, etologico' ${ }^{1}$ o psicoanalitico. Psicanalisti ed etologi vedono il comportamento aggressivo come parte integrante del DNA dell'uomo, dunque impossibile da sopprimere in quanto condizione biologica immutabile; diversamente, per la sociologia e la psicologia, non si può che tentare di contenere e dominare tale condotta mediante il processo di socializzazione, adeguandosi a determinate norme sociali.

Nel linguaggio comune, quando si parla di aggressività si fa riferimento a una predisposizione rispetto a un comportamento orientato all'affermazione di sé e al trionfo sull'avversario. Attraverso un'attività di competizione che può essere intesa come costruttiva oppure negativa, l'aggressività può essere percepita come fonte di vita e, allo stesso tempo, di distruzione: è una forza dinamica che grava in senso positivo sulle relazioni sociali tra $\mathrm{i}$ singoli quando mira alla riuscita personale, al successo delle proprie capacità, al miglioramento della qualità della vita; al contrario, è vista come un impulso negativo quando origina conflittualità e si esprime in eventi violenti, dalla forma più estrema con l'annientamento fisico del nemico ad altre forme quali la sottomissione o lo sfruttamento dell'altro, vittima della paura, personalità priva di adeguata forza morale e autostima. (Pellegrino A., 2018). ${ }^{2}$
Questo concetto viene evidenziato anche dall'etimologia stessa del termine "aggressione" che deriva dal latino aggredior (ad+ gradior $=$ vado, cammino, mi avvicino, entro in contatto ecc.). Evidente, dunque, la componente relazionale che l'aggressività contiene, si intende il rapportarsi a qualcuno o qualcosa agendo in maniera benigna e quindi col fine di accattivarsi, assicurarsi un

"etologìa s. f. [comp. di eto- e -logia]. - 1. non com. a. Studio dei caratteri e dei costumi di un popolo. b. Parte della psicologia differenziale, detta più spesso caratterologia, che determina e classifica i varî tipi di carattere, ne ricerca le cause e ne studia le anomalie. 2. Ramo della biologia che studia il comportamento animale, inteso sia come studio delle azioni di un singolo individuo, sia come studio dei rapporti tra gli individui di una stessa specie, sia come studio delle interazioni tra specie differenti. E. comparata, studio parallelo del comportamento di specie diverse, interessante soprattutto perché ha consentito la comprensione dell'evoluzione del comportamento." (www.treccani.it). Gli etologi credono che "ogni apprendimento trovi il suo fondamento in un sistema inserito nel codice genetico della specie animale che induce l'organismo a moduli comportamentali indispensabili per la conservazione dell'individuo". (www.riservadelladuchessa.it)

2 Pellegrino A. (2018) - Violenza e società 2. Aggressività e natura umana - in: https://letteredallafacolta.univpm.it/aggressivita-e-societa/ 
favore; oppure l'avvicinarsi in modo ostile, perciò assalendo, danneggiando o accusando. ${ }^{3}$

"Parlare di aggressività non è semplice, trattandosi di un concetto polisemico e multifattoriale. I significati sono veramente molti, tanto che in letteratura troviamo più di 250 definizioni differenti di aggressività (Hare et al.,1983). ${ }^{4}$

Una fenomenica delle condotte aggressive tende a distinguere differenti tipologie (Attanasio, 2012) ${ }^{5}$ :

Un'aggressività attiva, nella quale l'individuo tenta di arrecare danno a un suo simile, mediante l'impiego della forza;

- Un'aggressività passiva, caratterizzata da atti di omissione (ad esempio il non prestare operazioni di aiuto verso chi si trova in uno stato di necessità);

- Un'aggressività diretta, nella quale si arreca danno con modalità mirata, in cui si utilizza il proprio corpo per arrecare sofferenza;

- Un'aggressività indiretta (ad esempio diffamare un soggetto per arrecargli danno)

- Un'aggressività autodiretta (in cui l'oggetto da aggredire diventa il sé);

- Un'aggressività eterodiretta verso oggetti o persone;

- Un'aggressività reattiva, provocata da un torto subito e alimentata dal sentimento di vendetta;

- Un'aggressività proattiva in cui la violenza, sia essa fisica o psicologica, viene programmata con una strategia studiata per nuocere all'altro."

L'aggressività viene presentata contrapponendosi alla nozione di violenza. Nella prima viene enfatizzato l'elemento istintuale mentre, con il termine violenza, si fa riferimento ad atteggiamenti antisociali, intenzionali e organizzati. Si può perciò distinguere un'aggressività semplice da una trasformata che in realtà si configura come violenza andando a sottolineare i risultati distruttivi dell'aggressività.

"La violenza è un atto o comportamento che faccia uso della forza fisica (con o senza l'impiego di armi o di altri mezzi d'offesa) per recare danno ad altri nella persona o nei beni o diritti. In senso più ampio, l'abuso della forza (rappresentata anche da sole parole o da sevizie morali, minacce, ricatti), come mezzo di costrizione, di oppressione, per obbligare cioè altri ad agire o a cedere contro la propria volontà.

Dalla violenza intesa come forma estrema di aggressione o come forma di coercizione e dominio, il termine è passato a significare ogni forma d'influenza, condizionamento e controllo delle potenzialità pratiche e intellettuali degli esseri umani. (...) L'indagine empirica sulla violenza come fatto sociale necessita di una specificazione dei criteri che permettono di classificare un atto come violento. Un primo criterio richiede che l'atto debba essere distruttivo di cose o persone, o tale da costituire minaccia di distruzione; un secondo criterio richiede che l'oggetto della distruzione debba avere un valore per la vittima o per la società in generale; un terzo criterio richiede la presenza di un attore, individuale o collettivo". 7

In questo elaborato inizieremo con incentrare il focus del discorso sugli indicatori e i segni predittivi di violenza e aggressività, e su quei fattori che influenzano le interazioni tra gli individui e che spingono l'essere umano ad agire mediante una condotta deviante e aggressiva; cercando di individuare quanto la componente sociale e quella individuale pesino sulle azioni dell'uomo, e perché e in quali casi dall'acquiescenza e il conformismo scaturiscano atti di violenza efferata.

Successivamente, diventa necessario un attento e accurato approfondimento relativamente a diversi casi studio di eventi recenti in cui si è manifestato l'impiego di un comportamento violento.

Si conclude l'intero elaborato con delle proposte di repressione e prevenzione della violenza, fornendo una visione completa di quelle che sono le nuove tecniche di approccio al fenomeno violento, delle innovative misure di prevenzione promozionale, attuali e funzionali allo scopo cautelare e precauzionale nella lotta alla devianza e alla criminalità.

\section{L'origine multifattoriale della violenza}

Il male è l'esercizio del potere. Questa è la chiave: si tratta di potere, di danneggiare intenzionalmente le persone dal punto di vista psicologico, ferirle fisicamente, distruggerne la morale, le idee e di commettere crimini contro l'umanità.

I sociologi, per decifrare l'origine della devianza, cercano sempre di capire e comprendere quello che c'è "dentro" una persona, una mela marcia e malvagia. Sì, le persone sono gli attori sul palcoscenico, ma bisogna sempre essere consapevoli della situazione. Chi è l'intero cast? Quali sono i costumi? C'è un direttore di scena?

$\mathrm{Ci}$ interessa sapere quali siano $\mathrm{i}$ fattori esterni che gravano attorno all'individuo, la mela marcia. Ed è questo il punto focale: il potere è nel sistema. Il sistema crea la situazione che corrompe gli individui, il sistema è il contesto legale, politico, economico e culturale. È qui che il potere diventa dei cattivi. Se si vuole cambiare una persona, bisogna cambiare la situazione, e per cambiarla bisogna sapere dove è il potere all'interno del sistema. È un'interazione dinamica dei vari fattori.

Prendendo in considerazione le teorie di alcuni studiosi che hanno analizzato l'utilizzo della violenza dall'età adolescenziale e studiato tale fenomeno mediante l'esame di alcuni casi clinici o tramite lo sfruttamento di ampi esempi statistici, è stato possibile osservare che alla base di una condotta violenta può esserci, appunto, un'interazione simultanea di diversi fattori e condizioni, vale a dire variabili di tipo genetico, socioculturale, neurologico, ambientale, ecc.

Potendo quindi attestare l'origine multifattoriale della violenza, diventa necessario approfondire tali elementi appena citati in modo da poter fornire un panorama globale, a 360 gradi, circa l'origine del comportamento violento.

\section{I fattori ereditari}

Non si può discutere sull'esistenza di un "gene" della delinquenza. La predisposizione al comportamento antisociale è frutto di un insieme di influenze ereditarie che agiscono mutuamente e in maniera multiforme, provocando di conseguenza una maggiore inclinazione verso una condotta deviante antisociale.

L'influsso ereditario con maggiori effetti sull'eredità biologica è quello che comprova la presenza di rischi genetici nei soggetti

\footnotetext{
3 Masala Carmelo, Preti Antonio, Petretto Donatella Rita (2002), L'aggressività, Psicologia e metodi di valutazione, Carocci Editori, Roma, p. 22.

Hare R.D. (1983) Psychopathy and violence. R. Hays, T.K Roberts, K.S. Solway (Eds.) (1981), Violence and the violent individual Attanasio S. (2012). Psicologia Sociale e Devianza, Lezione 11. www.ipsico.it www.treccani.it
} 
affetti da disturbo schizoide di personalità, caratterizzati da inadeguatezza psicosociale con forte distacco e isolamento; personalità incapaci di esprimere emozioni e di provare empatia, che tendono ad avere uno stato d'animo negativo dovuto anche allo sviluppo di una forma di ostilità verso i loro pari socialmente adatti. Questi soggetti sono molto impulsivi e inclini a commettere atti violenti, sono sadici in costante ricerca di rischi e sensazioni forti. ${ }^{8}$

Per quanto invece concerne l'aggressività, si conta uno scarso numero di pubblicazioni che comprovino la possibilità di trasmettere il comportamento aggressivo per mezzo del patrimonio genetico, conciossiacosaché una ricerca abbia effettivamente appurato una correlazione tra la condotta aggressiva nei bambini adottati e i padri biologici che soffrono di disturbi di personalità antisociale.

Un ulteriore studio è stato effettuato presso l'Università di Montreal. Gli esperti hanno condotto una valutazione psicosociale su 555 coppie di gemelli, di cui 223 monozigoti e 332 eterozigoti. ${ }^{9}$

"Avere a disposizione entrambe le tipologie di gemelli ha permesso di valutare se le differenze individuali rilevate fossero legate a fattori genetici o ambientali. Le condotte aggressive venivano registrate dagli insegnanti, per avere un punto di vista neutrale extra familiare, all' età di $6,7,9$, 10 e 12 anni. I ricercatori si sono concentrati sui comportamenti aggressivi proattivi e reattivi. Per aggressività proattiva si intende una forma di aggressività caratterizzata da comportamenti fisici e verbali finalizzati al dominio o all'ottenimento di un vantaggio personale a scapito degli altri. Per aggressività reattiva viene fatto riferimento a quel tipo di aggressività caratterizzata da una risposta di difesa quando viene percepita una minaccia.

La valutazione dei risultati ha fatto emergere che all'età di 6 anni si osservano nei bambini entrambi i tipi di aggressività, reattiva e proattiva, condividendo quasi totalmente gli stessi fattori genetici. Con la crescita si osserva che il comportamento aggressivo tende a decrescere, giungendo alla conclusione che tra i 6 ed i 12 anni i fattori ambientali hanno un peso maggiore rispetto alla componente genetica.

In quest'ottica l'aggressività viene considerata come un elemento físiologico e costitutivo dello sviluppo psico-sociale del bambino. Con la crescita il bambino impara a gestire meglio le proprie emozioni riuscendo ad adottare modalità di comunicazione più idonee al buon funzionamento sociale, percorso che dovrebbe proseguire e affinarsi sempre più nell'età adulta". ${ }^{10}$

\section{I fattori neurologici}

Oltre a quanto già detto, un ulteriore elemento che tende a intensificare le manifestazioni aggressive della persona va riconosciuto nella sua irritabilità neurofisiologica; difatti i soggetti caratterizzati da tratti di personalità borderline o narcisistici, stati psicopatologici o soggetti che hanno subito danni o deterioramenti cerebrali nell'infanzia, così come chi abusa di sostanze stupefacenti o alcool, sono maggiormente esposti e proclivi a tendenze violente. Questi individui appena citati hanno particolare disposizione verso manifestazioni violente cagionate dalla sussistenza di disfunzioni cerebrali in zone specifiche della corteccia, preposte a pulsioni e centri delle decisioni sociali. Ciò provoca l'abbassamento della tolleranza alla tensione psichica determinata da un alto grado di frustrazione, generando risposte allo stress eccessivamente violente.

Celebre è il caso di Phineas Gage, un operaio caposquadra nell'ambito delle costruzioni ferroviarie negli Stati Uniti che miracolosamente sopravvisse a un grave incidente sul luogo di lavoro: è il 13 settembre del 1848 quando, a causa di un'esplosione acciden- tale, una barra di ferro gli trapassò il cranio, danneggiandogli il lobo frontale sinistro del cervello. Quell'avvenimento cambiò la vita dell'uomo che perse il controllo della sua mente e iniziò a delirare. Quello di Gage "fu il primo caso a suggerire il ruolo del cervello nel determinare la personalità e che il danno a parti specifiche del cervello potesse indurre cambiamenti di personalità specifici." ${ }^{11}$ Harlow, il dottore che curò Gage nell'arco di tutto il periodo di convalescenza, fu l'unico a trascrivere le testimonianze di conoscenti circa l'alterazione della personalità di Gage da prima e a dopo l'incidente, Harlow scrisse:

"L'equilibrio tra le sue facoltà intellettuali e le propensioni animali sembra essere stato distrutto. È blasfemo, irriverente, volgare, come non era mai stato prima dell'incidente. È capriccioso e sconclusionato e non accetta consigli. Infantile nelle sue capacità intellettuali e manifestazioni, ha le passioni animali di un uomo forte. Amici e conoscenti dicono che non è più Phineas Gage".

I danni alla corteccia cerebrale costituiscono quindi un fattore che predispone alla condotta violenta quando presenti sin dall'adolescenza. Gli adolescenti che hanno subito danneggiamenti ai lobi frontali prima dello sviluppo di un self-control adatto e conforme, sono più propensi alla concretizzazione di un'indole violenta e senza inibizioni rispetto a coloro che hanno sostenuto questi danni in un periodo tardivo.

Secondo diversi studi (Goldstein et al., 2005; Ulzen \& Hamilton, 1998) la prevalenza della malattia mentale nei delinquenti minorili è significativamente più alta che nella popolazione adolescenziale generale. Infatti, Goldstein et al. (2005) hanno definito i disturbi mentali "il fattore di rischio trascurato nella delinquenza giovanile" (p. 85). ${ }^{12}$ Non sorprende che Eppright et al. $(1993)^{13}$ abbiano provato che 1' $87 \%$ di un gruppo di minori autori di reato soddisfaceva i criteri diagnostici per il disturbo della condotta. Inoltre, è stato anche riscontrato che i giovani autori di reati minorili hanno sperimentato una varietà di disturbi mentali e di comorbilità. ${ }^{14}$ Sulla base della sua esperienza di valutazione dei giovani autori di reati, Abram et al. (2002) $)^{15}$ ha incoraggiato i professionisti della salute mentale di prevedere che almeno 1 giovane su 10 presso le strutture di giustizia minorile avrà sia un grave disturbo mentale (psicosi, episodi maniacali o depressivi) sia un disturbo da abuso di sostanze. ${ }^{16}$

8 Rutter M., Giller H. and Hagell A. (1998) Antisocial Behavior By Young People. Cambridge University Press.

9 Paquin, S., Lacourse, E., Brendgen, M., Vitaro, F., Dionne, G., Tremblay, R. E., Boivin, M. (2017). Heterogeneity in the development of proactive and reactive aggression in childhood: Common and specific genetic-environmental factors. PloS One 12, e0188730.

10 www.ipsico.it

11 Alfiero A. (2019) - Phineas Gage: danni al cervello e comportamento. https://www.thedifferentgroup.com/2019/12/22/phineas-gage/

12 K. Heilbrun, N.E. Sevin Goldstein, R, E, Redding (2005), Juvenile Delinquency: Prevention, assessment and intervention, OUP USA.

13 Eppright T.D, Kashani J.H, Robison B.D, Reid J.C, (2003), Comorbidity of conduct disorder and personality disorders in an incarcerated juvenile population. https:/ajp.psychiatryonline.org/doi/abs/10.1176/ajp. 150.8.1233

14 Comorbilità: in medicina, la presenza contemporanea nello stesso soggetto di due o più malattie. www.treccani.it

15 Teplin L.A., Abram K.M., McCLelland G.M., Dulcan M.K., Mericle A.A. (2002), Psychiatric disorders in youth juvenile detention, Arch Gen Psychiatry, 59:1133-43.

16 Tradotto da www.etd.ohiolink.edu 


\section{I fattori ambientali}

Come abbiamo già detto in precedenza, una convergenza di vari fattori biologici, emotivi, sociali e ambientali può contribuire alla stimolazione di comportamenti violenti, acting-out antisociali e disturbi della condotta, specialmente a causa dell'interazione tra quelle vulnerabilità emotivo/cognitive interne e i fattori ambientali negativi (Lewis, 1990). ${ }^{17}$

Il bambino, a contatto con il mondo esterno, entra in relazione con un ambiente saturo di modelli di riferimento che incitano e spesso giustificano la violenza; una realtà povera in cui sono particolarmente frequenti esempi di comportamenti violenti all'interno di una comunità, modelli che possono essere in larga parte appresi.

Al fine di comprendere il fenomeno della violenza, l'elemento familiare diviene essenziale in quanto costituisce uno degli agenti principali nello stabilire l'influenza dell'ambiente sulla condotta, invero si verifica un incremento della percentuale dei rischi di comportamenti violenti laddove maggiori sono gli aspetti ambientali negativi e tossici all'interno del contesto familiare.

"Numerose ricerche hanno messo in luce una forte correla-

zione tra particolari aspetti dell'ambiente familiare e comportamenti aggressivi nei bambini e negli adolescenti, in particolare un basso livello socioeconomico (Sameroff, 1987), ${ }^{18}$ l'essere genitori single (Webster-Stratton, 1990), ${ }^{19}$ alti livelli di stress e depressione materna (Campbell, $1990)^{20}$ e l'esposizione a violenza fisica e psicologica (Juoriles et al., 1980), ${ }^{21}$, contribuirebbero alla formazione di condotte distruttive. Spesso si tratta di genitori con personalità antisociali, che elargiscono al bambino dure punizioni fisiche, non forniscono un'adeguata supervisione e sono poco coinvolti e presenti nella vita del figlio". ${ }^{22}$

Studi e ricerche dimostrano come il maltrattamento fisico, verbale e psicologico sia la fonte che cagioni la condotta violenta nei bambini. Wekerle e Wolfe, nel 1998, hanno analizzato diversi casi di violenza giovanile, giungendo alla conclusione che circa il 13$18 \%$ dei comportamenti devianti nell'adolescenza, che siano essi di tipo sessuale, psicologico o verbale, hanno origine dal maltrattamento genitoriale avvenuto durante l'infanzia. È stato clinicamente dimostrato come un maltrattamento precoce comprometta lo sviluppo cerebrale, specialmente del sistema limbico, danneggiando il cervello in una forma paragonabile a quella derivante da anormalità genetiche e neurologiche (Van Der Kolk et al. 1985). ${ }^{23}$

Violenza e criminalità che "affondano le proprie radici in un fallimento educativo, il quale non rappresenta solo l'incapacità di fornire ai giovani le adeguate competenze cognitive, ma anche l'incapacità di renderli soggetti dotati di valori umani e civici. Quando determinati soggetti sociali si sentono privi di valori fondanti nei contesti dove vivono, quando si sentono ignorati e non hanno la possibilità d'intravedere un futuro in cui dimostrare il proprio valore e le proprie capacità, l'esercizio della violenza diventa un modo per dimostrare la propria superiorità rispetto ai coetanei e agli adulti. Esistono precise responsabilità politiche e sociali di fronte alla povertà, ai problemi delle famiglie, alla debolezza degli interventi preventivi, all'abbandono scolastico e formativo, perché gli interventi riparatori sono deboli e manca un coordinamento nazionale, regionale locale. Non si ritiene possibile che le pulsioni violente possano essere controllate attraverso un processo di socializzazione che responsabilizzi gli individui, sviluppi l'empatia all'interno del gruppo sociale, educhi a controllare qualunque comportamento violento". ${ }^{24}$

Un'ulteriore fonte di apprendimento sono i mass media. Televisione, cinema, fumetti e stampa offrono un alto tasso di contenuti di natura violenta che potrebbero, di conseguenza, andare ad accrescere e indurre comportamenti devianti nella realtà vissuta. È assodato che alcuni soggetti sono più inclini a reagire con violenza se sottoposti a messaggi violenti a differenza di altri, probabilmente meno narcisisti e meno angosciati da un eventuale fallimento personale. L'esposizione a tematiche di stampo violento o ad atteggiamenti aggressivi può avere, nei lunghi periodi, un effetto inconscio e non immediato, generando quindi una desensibilizzazione emotiva rispetto alla violenza nella vita reale, un'impassibilità nei confronti del dolore altrui, producendo una tendenza a reputare come prevedibili e ineluttabili alcuni eventi criminosi, si ha quindi una tolleranza passiva del male attraverso l'inazione o l'indifferenza. Un'altra conseguenza negativa della trasmissione di programmi violenti o di notizie di cronaca nera è l'aumento del timore di divenire bersaglio di manifestazioni violente, inducendo a provare un sentimento di diffidenza nei confronti del prossimo.

\section{I fattori culturali}

Unitamente ai fattori finora delineati e identificati, sussistono ulteriori fattori di matrice socioculturale che concernono la peculiarità e l'iterazione della violenza.

Le origini del comportamento aggressivo, oltre alle componenti neuro-fisiologiche, si fanno risalire anche alle condizioni culturali. Difatti, la scarica aggressiva si può descrivere "come un comportamento socio-indotto o inibito sulla base dei modelli sociali preposti", ${ }^{25}$, introducendo così una dimensione gruppale oltre a quella unicamente individuale.

L'aggressività dipende da una serie di norme e regole più o meno implicite, che generano sia la tendenza aggressiva, ma anche le modalità per poterla esprimere sia dentro al gruppo che fuori: diviene quindi una condizione modulata dal contesto socioculturale che può attivarla o disattivarla attraverso dei meccanismi sociali che facilitano la deresponsabilizzazione dell'individuo. ${ }^{26}$

"La società e il contesto assumono, per questo, forte valenza di controllo sul comportamento aggressivo dell'individuo, che emerge come debole sul controllo delle proprie azioni, laddove gli si offre un contesto che lo solleva o lo esonera dei processi di autocolpevolizzazione". ${ }^{27}$

17 Lewis, D. O. (1990). Neuropsychiatric and experiential correlates of violent juvenile delinquency. Neuropsychology Review, 1:125-36.

18 Sameroff, A.J., Seifer, R., Zax, M., Barocas, R. (1987). Early indicator of developmental risk: Rochester Longitudinal Study. Schizophrenia Bull 13:389-94.

19 Webster-Stratton, C. (1990). Stress: a potential disrupter of parent perceptions and family interactions. J Clin Child Psychol 19:302-12.

20 Campbell, S. B. (1990). Behavior problems in preschool children: Clinical and developmental issues. New York: Guilford Press.

21 Jouriles, E.N., Murphy, C.M., O’Leary K. D. (1989). Intersposual aggression, marital discord, and child problems. J Consult Clin Psychol $57: 453-5$

22 www.stateofmind.it

23 Van der Kolk, B., Greenberg, M., Boyd, H., Krystal, J. (1985). Inescapable shock, neurotransmitters, and addiction to trauma: Toward a psychobiology of post traumatic stress. Biol Psychiatry 20:314-25.

24 Pellegrino A. (2018), La violenza, i nuovi media e i "nativi digitali”. https://letteredallafacolta.univpm.it/violenza-societa-5/

25 Caponeri P., Il comportamento aggressivo nelle teorie psicoanalitiche. http://www.pierocaponeri.com/consulenza-psicologica/comportamento-aggressivo-nelle-teorie-psicoanalitiche/

26 ibidem

27 ibidem 
Un rifermento culturale particolarmente tipico potrebbe essere la caratterizzazione violenta di certe società, la civiltà americana per esempio, fin dalle sue origini attraversata da una fascinazione relativa agli eroi violenti e all'ammirazione a tratti perversa per i serial killers. Questa preoccupazione si esprime nel considerare l'enormità di armi da fuoco a disposizione e accessibili a chiunque.

\section{In cosa sfocia l'aggressività: analisi di casi recenti}

Portiamo ora alla luce alcuni recenti casi di cronaca nera che hanno scosso nel profondo le nostre coscienze.

L'omicidio è un atto atroce e disumano, trattare questo genere di avvenimenti non è mai una cosa che si riesce a fare con semplicità.

"Gli animali uccidono per fame e per istinto, mentre $i$ cosiddetti uomini d'onore, che a differenza delle bestie possono pensare, uccidono per odio e fame di potere."

Luigi Garlando

\section{L’omicidio di Willy Monteiro Duarte}

Willy Monteiro Duarte, un ragazzo di soli 21 anni, è la vittima "martirizzata" di questo brutale crimine avvenuto nella notte tra il 5 e il 6 settembre 2020, nella città di Colleferro.

\section{La ricostruzione dell'omicidio}

Willy è stato oggetto di una serie di feroci percosse inflitte da un gruppo di quattro ragazzi tra i 22 e i 26 anni di età, che ne hanno causato un grave shock traumatico e il conseguente arresto cardiaco.

Il giovane ragazzo era intervenuto in maniera pacifica e raziocinante tentando di porre fine a una lite scoppiata davanti a un locale, per difendere un amico finito nel mirino e invischiato in questa discussione scaturita da motivazioni molto più che banali. L'avversario decise di chiamare i "soccorsi" ed è qui che interviene il gruppo violento, quasi animalesco, un'orda selvaggia di quattro ragazzi che hanno rivolto tutta la loro rabbia e aggressività verso Willy, un ragazzo italiano di seconda generazione, nato da genitori di origini capoverdiane, gracile e dall'animo buono, a differenza del branco dei quattro accusati, due dei quali esperti di arti marziali miste.

Non è stata una rissa, ma si può definire un vero e proprio pestaggio, una spedizione punitiva contro il povero Willy, che non aveva un branco, e questo gli è stato fatale.

I quattro indiziati, i fratelli Gabriele e Marco Bianchi, insieme a Francesco Belleggia (che ha ottenuto gli arresti domiciliari) e Mario Pincarelli, sono stati arrestati in flagranza e inizialmente accusati di omicidio preterintenzionale ${ }^{28}$ in concorso, ${ }^{29}$ poi di omicidio volontario aggravato da futili motivi. ${ }^{30}$

La sproporzione fisica tra vittima e aggressori, insieme alla dinamica da linciaggio e la componente del colore della pelle di Willy, hanno fatto inizialmente pensare a un possibile crimine incitato dall'odio razziale, a un background da militanti neofascisti; ma ad oggi non è possibile sostenere con certezza tale ipotesi in quanto ne mancano gli elementi a favore. È perciò opportuno rimanere cauti nel giudizio. Non è possibile parlare di razzismo in assenza di una chiara motivazione ideologica, o di precedenti, o di un'attestata frequentazione da parte degli aggressori di ambienti esplicitamente razzisti che potrebbero averli condizionati. Il "movente razziale" è fatto ricondurre unicamente all'azione individuale del soggetto, serve perciò un'esplicita ideologia razzista, non viene pertanto considerato il fatto che il razzismo sia un atto strutturale che opera su un piano di sistema.
In questa vicenda sono presenti numerosi punti oscuri.

Cosa c'è alla base di una così barbara azione violenta? Da cosa nasce? Se entrambi i fratelli Bianchi sono stati coinvolti in una numerosa serie di risse negli ultimi tre anni, perché nonostante $\mathrm{i}$ precedenti non era scattato alcun tipo di provvedimento nei loro confronti? Marco è stato protagonista di ben cinque episodi rissosi, e non solo, aveva anche precedenti per spaccio, lesioni e minaccia. Perché sono arrivati ad agire in questo modo? Quale sottocultura ha influito?

Primo elemento di cui dobbiamo tenere conto è la zona di provenienza dei membri di quel gruppo/branco violento, originari della vicina cittadina di Artena, un centro abitato al di fuori della modernità e del caos della Capitale, rappresentato in maniera idilliaca da chi lo vuole far passare per quello che nella realtà non è. Artena, in seguito al recente triste caso del giovane Willy, viene descritto attraverso le immagini mediatiche, come un coacervo di criminalità e malavita, un covo di banditi, spacciatori e aggressori che vivono nel lusso. È regno di caos e disordine morale.

È una realtà di provincia che sta andando incontro a una deriva sociale, a una chiusura individualistica e conformista creando una sola e unica strada che non porta a un campo di scelte possibili dell'esistenza, bensì guida i suoi giovani verso una carriera criminale, attratti da un'ossessione folle per profumo del denaro facile, dalla necessità di mantenere un certo tenore di vita. In un paese in cui la violenza è percepita come regola di vita, il fare la cosa giusta diventa una debolezza.

Tutti i giovani cittadini di Artena, Paliano, Cori e Colleferro hanno questa visione del mondo e della vita; adorano seminare il terrore, risse e pestaggi sono all'ordine del giorno.

In questo caso sono cruciali le parole del presidente del Circolo Arci della stessa cittadina di Artena, Mino Massimei:

"Per un ragazzo qui ad Artena è più facile intraprendere un'attività di spaccio, anche piccola, che tentare un tirocinio formativo o un primo contratto di lavoro subordinato, perché tra l'altro si guadagna di più. Poi un segmento di popolazione giovanile è affascinato dalla vecchia criminalità di 5060 enni e da un immaginario legato al culto della forza". ${ }^{31}$

28 'L'omicidio preterintenzionale è la fattispecie di reato disciplinata dall'art. 584, c.p., il quale definisce come colpevole "chiunque, con atti diretti a commettere uno dei delitti preveduti dagli articoli 581 e 582, cagiona la morte di un uomo, è punito con la reclusione da dieci a diciotto anni." https://www.brocardi.it/codice-penale/libro-secondo/titolo-xii/capoi/art584.html

È fondamentale e necessario che la morte sia avvenuta a seguito di atti diretti a percuotere o ledere qualcuno e che perciò esista il nesso di casualità tra azione prevista ed evento letale. La condotta si indentifica con le percosse (art. 581 c.p) o le lesioni (art. 582 c.p), ma non è necessario che questi vengano consumati per costituire reato; infatti l'evento letale deve essere il prodotto di una specifica situazione di pericolo ricreata dal reo tramite la sua condotta intenzionale. https:/www.altalex.com/documents/altalexpedia/2020/03/18/omicidio-preterintenzionale

29 È sufficiente la dimostrazione di concorso che può essere sia di tipo morale che materiale dei diversi individui attivi nell'azione senza che vi sia obbligatoriamente la sussistenza della volontà di uccidere, bensì è necessario un evidente nesso di casualità. https://www.altalex.com/documents/ altalexpedia/2020/03/18/omicidio-preterintenzionale

30 Si considerano le cosiddette "circostanze aggravanti" quegli elementi che sono accidentali e irrilevanti per la sussistenza del reato, ma che costituiscono una maggiore gravità e perciò prevedono un conseguente aumento della pena. https:/www.avvocatofrancescodandria.it/omicidioaggravato/

31 Pacoletti M. (2020), Willy Monteiro Duarte e la terribile normalità di una morte. https://www.valigiablu.it/willy-monteiro-duarte-omicidio-colleferro/ 
E forse qui possiamo ricollegarci a quelle teorie sociologiche della scuola di Chicago, le teorie sottoculturali, che considerano la delinquenza come l'espressione collettiva dei problemi di una determinata comunità.

Diventa perciò necessario anzitutto citare la teoria dell'anomia $^{32}$ di Durkheim, ${ }^{33}$ dalla prospettiva interpretativa di Merton ${ }^{34}$ che vedeva l'anomia come

"la conseguenza non voluta di un divario strutturale tra fini e mezzi. In una società che dà troppa importanza al successo personale, al raggiungimento di traguardi cui tutti dovrebbero aspirare ("il sogno americano"), e che tuttavia possiede una struttura che non offre a tutti uguali opportunità o uguali mezzi per raggiungere questi traguardi, si determina una tensione permanente. La risposta più comune a questa situazione sarà sempre il conformismo, cioè 1' accettazione dei mezzi legittimi e culturalmente prescritti (come l'impegno individuale), nonché dei traguardi approvati. Ma esistono anche adattamenti devianti, di cui i più importanti sono l'innovazione e la rinuncia. L'innovazione consiste nel perseguire i fini culturali prescritti (in particolare il successo materiale), facendo pero uso di mezzi illegittimi (per es. il furto, la frode, la violenza); la rinuncia (una categoria di comportamenti in cui Merton includeva gli " adattamenti devianti" come il suicidio, la malattia mentale e la tossicomania) consiste nel rifiuto sia dei fini prescritti sia dei mezzi convenzionali". 35

Questa concezione ebbe come conseguenza lo sviluppo delle teorie delle subculture che si focalizzarono soprattutto sulla delinquenza giovanile e si basavano sul presupposto che la delinquenza e la devianza rappresentassero l'unica soluzione culturale condivisa alle problematiche strutturali.

In questo caso diventa perciò essenziale il contributo dei testimoni per l'accertamento di quello che è la verità in sede processuale, ma in una realtà come quella di Artena e degli altri paesi limitrofi, le persone potrebbero avvertire un clima di paura, intimidazioni, omertà, perciò diviene molto più semplice tacere pur avendo l'opportunità di fare la differenza.

"Willy è stato assalito e picchiato a morte da 5 ragazzi di corporatura gigante e palestrati che erano il doppio di lui. Penso che nei suoi ultimi minuti di vita, preso da paura e dolore lancinante abbia urlato e cercato aiuto fino all'ultimo respiro: io non mi meraviglio della gente che non è intervenuta, conosco bene il mondo dove viviamo, ma mi spiegate come è possibile che tutto questo sia successo nemmeno a 10 metri di distanza dalla caserma dei carabinieri? Tutti devono sapere che se nel 2020 ti stanno picchiando a morte con calci e pugni, esattamente sotto la caserma, nessuno interverrà, nemmeno chi è pagato per farlo! C'è una vera e propria anarchia e tutto questo in piazza di fronte alla caserma". ${ }^{36}$

Un altro elemento fonte di dubbi e sospetti è la mancata tempestività nei soccorsi; difatti sono passati ben 40 minuti prima dell'arrivo dell'ambulanza in seguito alle nove diverse chiamate effettuate. Il ragazzo è morto a soli 250 metri dal Pronto Soccorso e a 15 metri dalla caserma dei carabinieri. Willy poteva essere salvato?

La Stampa ha ricostruito l'andamento dell'evento, arrivando a concludere che l'unica ambulanza di Colleferro quella sera era già impiegata in un altro intervento, perciò non è stato possibile agire in maniera tempestiva, ma è stato necessario chiamare una seconda ambulanza che si trovava a Montelanico, distante circa quindici chilometri da Colleferro. Questo spiega l'enorme ritardo nel prestare soccorso.

Un altro caso analogo alla vicenda di Willy Monteiro Duarte è quello avvenuto due anni fa, il 6 ottobre del 2018, a Foggia. Donato
Monopoli, un ragazzo di 25 anni di Cerignola, è morto nelle stesse identiche dinamiche di Willy, in seguito a una violenta aggressione avvenuta in un locale di Foggia per mano di due suoi coetanei, Michele Verderosa e Francesco Pio Stallone. Il giovane, in seguito a un'emorragia al capo e a quattro diversi interventi, è deceduto dopo ben sette mesi di agonia in coma farmacologico, mentre i due colpevoli, pochi giorni dopo l'avvenuta scomparsa, sono riusciti a ottenere i domiciliari e dall'aprile 2019 girano liberi con obbligo di soggiorno a Foggia. Gli aggressori, quindi, furono inizialmente accusati di omicidio aggravato da futili motivi, in quanto si ipotizzava che alla base del pestaggio ci fosse un accidentale spintone tra Donato e M. Verderosa, ma dopo solo 6 mesi di carcere vennero posti ai domiciliari e successivamente liberati nell'aprile 2019. Il capo d'accusa è stato perciò tramutato: da omicidio volontario aggravato $i$ due indagati sono stati imputati per reato di omicidio ${ }^{37}$

"Lui è Donato Monopoli, ma la sua morte non fa audience" sono le parole scritte da uno dei tanti amici e parenti che aggiornano quotidianamente la pagina social "Giustizia per Donato".

Perché il caso di Donato non ha riscosso tanto clamore mediatico quanto quello destato dalla vicenda di Willy? È aperto lo sfogo sui social dove conoscenti, così come persone esterne ai fatti, si chiedono come mai Donato e tutti gli altri ragazzi morti nelle stesse condizioni sono passati in secondo piano. Esiste distinzione tra morte e morte?

Sembra brutto dirlo, ma forse è proprio il colore della pelle di Willy che ha influito sull'enorme apporto dei media sulla vicenda. Difatti, in questo periodo storico, stiamo vivendo in un'epoca caratterizzata da migliaia di proteste, rivolte razziali, ondate di ribellioni a livello internazionale nella lotta per l'uguaglianza razziale. Willy era un ragazzo di origini capoverdiane, perciò, in un periodo come questo, credo che la stampa italiana, in seguito alle numerose vicende sulla scena internazionale e al famoso movimento attivista Black Lives Matter, ${ }^{38}$ abbia voluto mettere in luce, sotto i riflettori dei media nazionali e magari globali, un ennesimo caso di violenza contro la comunità afroamericana. Per fare audience, perché è di questo che si parla. Perciò ci si chiede se diventare un caso mediatico sia quello che serva per ottenere giustizia in Italia.

32 Il famoso termine durkheimiano "anomia" si riferiva alla caratteristica dissociazione, nella società moderna, dell'individualità dalla coscienza collettiva. I desideri individuali, che emergono dal loro 'abisso insaziabile e senza fondo", non sono sufficientemente regolati o controllati. L'anomia era lo stato di mancanza di norme prodotto dalla rapida fuoriuscita dalla società tradizionale, ed esacerbato dalle crisi sociali ed economiche. https://2018.aulaweb.unige.it/pluginfile.php/234981/mod_resource/content $/ 2 /$ S.\%20Cohen $\% 20-\% 20$ sociologia $\% 20$ della\%20devianza.pdf

33 Émile Durkheim, famoso sociologo francese, è stato uno dei padri fondatori della sociologia (1858-1917).

34 Robert K. Merton, sociologo statunitense e uno dei principali esponenti della scuola funzionalista, scuola di pensiero americana che successivamente si avvicinò molto al comportamentismo (1910-2003).

35 Enciclopedia italiana Treccani.it, V Appendice, Cohen S., sociologia della devianza. https://2018.aulaweb.unige.it/pluginfile.php/234981/ mod_resource/content/2/S.\%20Cohen $\% 20-\% 20$ sociologia $\% 20$ della $\% 20$ devianza.pdf

36 Testimonianza di un giovane della zona raccolta da www.iene.it

37 Redazione de "L'Immediato" (2019), Ucciso di botte in discoteca a Foggia. "Schifati dalla giustizia, i due aggressori girano liberi". https://www.immediato.net/2019/05/08/ucciso-di-botte-in-discoteca-a-foggia-schifati-da-giustizia-i-due-aggressori-girano-liberi/

38 Letteralmente "le vite dei neri contano". BLM "è un movimento nato negli Stati Uniti impegnato nella lotta contro la discriminazione razziale e gli abusi da parte della polizia nei confronti dei cittadini appartenenti alla comunità afroamericana." www.open.online.it 
In un post sulla pagina dedicata a Donato qualcuno scrive: "Donato, ad oggi, non ha avuto nessun tipo di giustizia, nessuno ne parla, nessuno ha telefonato ai piani alti..." e ancora

"nessuno gli ha dedicato giorni di programmi tv, nessuno ha parlato del suo caso. Certo, all'inizio si, ma oggi? Come funziona? Donato e tutti gli altri ragazzi morti come lui, sono passati in secondo piano? No, non è così che dovrebbe funzionare. La giustizia deve arrivare per tutti, bisogna parlare di tutte le vittime, bisogna aiutare tutte le famiglie". 39

\section{Il duplice omicidio a Lecce - Eleonora Manta e Daniele De Santis}

\section{La ricostruzione dell'omicidio}

Il secondo caso preso in esame è invece datato 21 settembre 2020, quando intorno alle 21.30, un uomo e una donna sono stati accoltellati a morte sulle scale di una palazzina nei pressi della stazione ferroviaria di Lecce.

Le vittime di questa tragedia sono Daniele De Santis e la fidanzata Eleonora Manta, rispettivamente 33 e 30 anni, deceduti per emorragia a causa delle profonde lacerazioni inflittegli da un uomo che è stato successivamente visto fuggire da alcuni condomini: un uomo alto, vestito di nero, con guanti di pelle e passamontagna abbassato per coprirsi il volto e non rendersi riconoscibile. Questi elementi, insieme a un biglietto recuperato nel cortile esterno del palazzo, in cui vi era segnato il percorso da seguire per evitare le telecamere del vicinato e indubbiamente appartenuto al presunto assassino, sono dettagli significativi che hanno subito fatto pensare a un gesto premeditato. Il fascicolo è perciò per duplice omicidio aggravato dalla premeditazione. ${ }^{40}$

Si è presto seguita la pista del reato passionale in quanto, a seguito di una prima autopsia eseguita sul corpo inerme di Eleonora, si è immediatamente notato un estremo accanimento nei suoi confronti: 35 coltellate contro le 25 riscontrate sul corpo di Daniele. ${ }^{41}$

Dopo una settimana di indagini eseguite con rapidità e concitazione, è stata definitivamente confermata l'identità dell'aggressore: la polizia ha fermato il colpevole di questo crimine efferato, un ex coinquilino della coppia e studente di scienze infermieristiche, un ragazzo di soli 21 anni originario di Casarano, Antonio De Marco. Essenziale per l'identificazione è stato il famoso bigliettino insanguinato reperito all'esterno dell'abitazione, che ha permesso il riconoscimento della grafia dell'aggressore grazie al confronto effettuato con quella sui documenti custoditi al Comune e presso la prefettura di Lecce.

Il procuratore De Catris, incaricato dell'indagine, ha spiegato attraverso quali modalità è avvenuta la ricostruzione delle dinamiche dell'omicidio:

'Siamo davanti a una fortissima premeditazione confermata dall'ispezione che il soggetto ha fatto nei giorni precedenti nella zona dove abitava la coppia e dal biglietto ritrovato, biglietto che contiene tanto lo studio del percorso da fare per evitare le telecamere che, purtroppo, le modalità di programmazione dell'omicidio, modalità che sarebbe stata preceduta anche da una attività cosiddetta prodromica al delitto." 42

Antonio De Marco, infatti, la sera dell'omicidio aveva con sé delle fascette da elettricista, con le quali aveva probabilmente intenzione di agire sui corpi delle vittime, immobilizzandoli con il fine di seviziarli e sottoporli a tortura. Il giovane aveva poi proget- tato di ripulire l'intera scena del crimine, lasciando solamente una scritta sul muro che avrebbe dovuto riportare un messaggio per l'intera città. È quindi ipotizzabile che l'intento dell'aggressore fosse quello di dare una rappresentazione per la collettività.

È ancora incerto il movente del delitto, sono tanti gli interrogativi che vi ruotano attorno.

Il giovane ha motivato l'assassinio confessando di aver agito perché "erano troppo felici" proseguendo "per questo mi è montata la rabbia. So di aver commesso una cavolata e di aver sbagliato gravemente."

Il comandante provinciale dell'arma dei carabinieri, Paolo Dembech, ha infine escluso il movente passionale: secondo quanto da lui riportato, le effettive cagioni del delitto devono essere ricercate in relazione al periodo di due mesi di convivenza con la coppia; l'assassino, infatti, un ragazzo "introverso, chiuso e con poche amicizie", ha espresso il suo fastidio destato dallo stato e dal sentimento felice delle vittime. Non sono però escluse altre ipotesi, come quella secondo la quale Antonio avrebbe agito spinto dal senso di rancore provato nei confronti della coppia che lo avrebbe "sfrattato" dalla stanza che Daniele gli aveva affittato nel loro stesso appartamento, sfratto avvenuto in seguito alle lamentele di Eleonora, a disagio con la presenza di Antonio, facendo così scaturire in lui un feroce senso di vendetta. Sul suo profilo social, De Marco ha inoltre pubblicato un post dal blog "universo psicologia" incentrato sulla questione del desiderio di vendetta, commentando "Un piatto da servire freddo...è vero che la vendetta non risolve il problema ma per pochi istanti ti senti soddisfatto", 43 concludendolo con due emoticon sorridenti.

Stando a quanto riportato nel provvedimento di fermo:

"L'azione è stata realizzata con spietatezza e totale assenza di ogni sentimento di pietà verso il prossimo"

Inoltre:

"nonostante le ripetute invocazioni a fermarsi urlate dalle vittime l'indagato proseguiva nell'azione meticolosamente programmata inseguendole per casa, raggiungendole all'esterno senza mai fermarsi. La condotta criminosa, estrinsecatasi nell'inflizione di un notevole numero di colpi inferti anche in parti non vitali (il volto di De Santis) e quindi non necessari per la consumazione del reato, appare sintomatico di un'indole particolarmente violenta, insensibile ad ogni richiamo umanitario".

E ancora:

"La sproporzione tra la motivazione del gesto (potrebbe avere avuto in precedenza una lite) e l'azione delittuosa è ulteriore elemento tale da fare ritenere che quest'ultima sia stata perpetrata per mero compiacimento sadico nel provo-

39 Dalla pagina Facebook "Giustizia per Donato" https://www.facebook.com/Michelemonopoli1973

40 Quotidiano.net (2020), Lecce, duplice omicidio. Ucciso l'arbitro Daniele De Santis e la sua compagna. https:/www.quotidiano.net/cronaca/duplice-omicidio-lecce-1.5533112

41 Spagnolo C. (2020), Fidanzati uccisi a Lecce, l'assassino mascherato si è accanito con violenza su Eleonora. https://bari.repubblica.it/cronaca/2020/09/23/news/coppia_uccisa_a_lecce_1_assassino_mascherato_si_e _accanito_con_violenza_su_eleonora-268287698/

42 Scognamiglio R. (2020), Preso il killer della "mattanza": "Conosceva Eleonora e Daniele". https://www.ilgiornale.it/news/cronache/coppia-uccisa-lecce-preso-lassassino-1893160.html

43 Spagnolo C. (2020), Fidanzati uccisi a Lecce, l'assassino mascherato si è accanito con violenza su Eleonora. https://bari.repubblica.it/cronaca/2020/09/23/news/coppia_uccisa_a_lecce_1_assassino_mascherato_si_e _accanito_con_violenza_su_eleonora-268287698/ 
care con le predette modalità la morte della giovane coppia. Non si spiega se non nella direzione di inquadrare l'azione in un contesto di macabra ritualità la presenza di oggetti non necessari a provocare la morte della giovane coppia (striscette, soda, ecc.). A tal riguardo giova altresì evidenziare come sul copricapo sia stata disegnata con un pennarello nero una bocca, quando ciò non risultava necessario all'economia del reato. Tali elementi unitariamente considerati fanno ritenere assai probabile il pericolo di recidiva in considerazione dell'estrema pericolosità dell'indagato". 44

Ognuna di queste dichiarazioni ci aiuta nella stesura del profilo psicologico del soggetto omicida.

Possiamo sicuramente definirlo come una personalità sadica, posta a un livello di funzionamento borderline e caratterizzata da un tipo di funzionamento del carattere con gravi distorsioni. Questo genere di persone, infatti, sono solite non provare alcun tipo rimorso o passione per il prossimo, sono capaci di infliggere torture con una calma quasi glaciale. Esenti da un qualsiasi turbamento interiore, organizzano e ricostruiscono il proprio scenario sadico con distacco, con ordine e criterio sconcertante, tenendo da conto ogni minimo particolare del loro piano spietato, senza sentirsi in colpa per la sofferenza inflitta. Il sadico prova estremo piacere nel provocare dolore alle sue vittime, vuole prolungare il più possibile la sottomissione di queste al suo controllo, perciò non sono rare torture e sevizie che non portano all'immediato decesso della vittima e, di conseguenza, del piacere stesso del sadico.

Nel PDM, ${ }^{45}$ questo disturbo è classificato come disturbo antisociale di personalità inerente alla psicopatia e al narcisismo maligno. ${ }^{46}$. Esistono perciò definiti criteri diagnostici che consentono la classificazione della persona sadica all'interno dei disturbi di personalità.

"Le caratteristiche del disturbo sadico di personalità sono così riassunte dal PDM (Psychodynamic Diagnostic Manual):

- Tensione/preoccupazione principale: patire umiliazioni/infliggere umiliazioni.

- Affetti principali: odio, disprezzo, piacere (godimento sadico).

- Credenza patogena caratteristica relativa a sé stessi: ho il diritto di infliggere dolore e umiliare le altre persone.

- Credenza patogena caratteristica relativa alle altre persone: gli altri esistono in quanto oggetti del mio dominio.

- Modi principali di difendersi: distacco, controllo onnipotente, ribaltamento, agiti." 47

Considerato il fatto che Eleonora non si sentisse a suo agio con la presenza di Antonio sotto il suo stesso tetto, possiamo probabilmente far ricondurre il suo senso di disagio proprio in relazione al soggetto sadico. Infatti, chi si relaziona con un individuo con tale disturbo, non è raro provare intimidazione e paura, una sensazione di malessere quasi viscerale.

Un ulteriore elemento che ci fa ipotizzare la personalità sadica di Antonio De Marco, è l'inquietante atteggiamento da lui assunto nei giorni seguenti al delitto. Infatti, Antonio avrebbe continuato a frequentare le lezioni in maniera regolare, così come sembra aver preso parte a una festa in tutta tranquillità, senza destare alcun sospetto. I carabinieri inoltre riportano come nel momento del prelievo e dell'arresto, Antonio non abbia mostrato nessun segno di pentimento, anzi, sarebbe scoppiato a ridere mostrandosi completamente distaccato e umanamente insensibile.

A questo proposito, per concludere, sono esemplari le parole dello psicologo statunitense, Paul Ekman:

"L'incapacità assurda di provare vergogna o sentirsi in colpa per i propri misfatti è considerato segno caratteristico dello psicopatico, se si estende a quasi tutti gli aspetti della vita".

\section{Conclusioni}

Pare tanto assurdo quanto irrazionale il fatto che le origini della devianza derivino proprio dall'intento e dalla volizione di "prevenire, punire o rieducare" di quelle stesse istituzioni preposte al controllo sociale che vanno invece a causarne il concretamento soprattutto attraverso i sistemi di definizione pubblica e stigmatizzazione. È la consecutiva presenza di una "reazione sociale" che risulta poi essere determinante per la costruzione della devianza.

Negli ultimi decenni si è a lungo discusso su quale fosse la migliore soluzione per la violenza e, accanto ai tradizionali approcci sanzionatori e segregativo-custodiali, sono nate nuove e più attuali prospettive in senso promozionale. Mentre i modelli tradizionali agiscono mediante una logica punitiva di carattere generale preventivo, con lo scopo di disincentivare il comportamento criminale esercitando una funzione deterrente e minacciando una punizione; i nuovi modelli promozionali presuppongono una migliore deterrenza attraverso il potenziamento del legame tra le risposte istituzionali e sociali e le risorse personali, relazionali e contestuali del soggetto stesso (De Leo, Patrizi, 2002). ${ }^{48}$ La custodia quale risposta a un determinato crimine ha funzione preventiva speciale, ovvero mira a disincentivare la recidiva del reo, ma non ha effettivamente nessun impatto nell'evitare l'insorgenza di tale comportamento.

"Sono tuttavia proprio le caratteristiche costitutive del fenomeno in oggetto che rendono estremamente difficile la produzione di consenso intorno a questo tema, trattandosi in primo luogo di un fenomeno socialmente costruito, che vede interagire in modo complesso e interdipendente i tre sistemi portanti che lo producono, quello dei comportamenti (individuali o gruppali), delle norme e delle reazioni sociali; in secondo luogo, di un fenomeno processuale, non ascrivibile linearmente a caratteristiche decontestualizzate, stabili e facilmente identificabili degli individui, dei gruppi o dei sistemi sociali; infine di un fenomeno profondamente radicato nei conflitti socioculturali e di interessi, capace di attivare forze e risposte sociali connotate da potenti valenze etico-morali." 49

44 Affariitaliani.it (2020), Omicidio Lecce, 21enne: "erano troppo felici, mi è salita la rabbia". https:/www.affaritaliani.it/cronache/omicidiolecce-2 1 enne-sbagliato-loro-troppo-felici-la-mia-una-cavolata696857.html?refresh_ce

45 Psychodynamic Diagnostic Manual (Manuale Diagnostico Psicodinamico) è un manuale contenente la classificazione di disturbi mentali e di personalità suddivisi al suo interno in quelli afferenti al livello borderline e quelli a livello nevrotico, offrendo perciò un quadro clinico completo della personalità sia di pazienti adulti che bambini. Il PDM, basato su un approccio di tipo non categoriale e con criteri diagnostici psicodinamici, permette inoltre di strutturare un conseguente progetto terapeutico. www.psychiatryonline.it

46 Termine coniato nel 1964 da Erich Fromm utilizzato per descrivere la condizione di una personalità caratterizzata da comportamenti ostili $\mathrm{e}$ antisociali. Questa persona manca di empatia e disumanizza qualsiasi scenario in cui si ritrova che sia esso di tipo familiare o lavorativo. Per Fromm questa condizione era la "quintessenza del male". www.lamenteemeravigliosa.it

47 Campisi M., Stile e disturbo sadico di personalità. https://campisipsicologo.com/disturbi/disturbo-sadico-di-personalita/

48 De Leo G., Patrizi P. (2002), Psicologia della devianza, Carocci.

49 Patrizi P., Psicologia della devianza e della criminalità. Teorie e modelli di intervento, Carocci editore. 
La prevenzione della devianza dovrebbe perciò essere di interesse prevalente rispetto a una gestione di tipo sanzionatorio, in quanto essenziale è l'intervento nel e con il sociale per intercettare, rilevare e accertare precocemente il problema, permettendo quindi una progettazione di eventuali soluzioni ante delictum. ${ }^{50}$ Bisogna perciò intervenire all'interno della comunità potenziandone la sicurezza sociale, adoperandosi per rafforzare la qualità del vivere comune, l'inclusione, la solidarietà sociale e infine, muovendosi verso lo sviluppo delle competenze soggettive e collettive che ne costituiscono l'innervatura.

Ma effettuare una riflessione in termini di prevenzione della criminalità significa poi spingersi verso un discorso molto più vasto $\mathrm{e}$ imperniato attorno alle politiche per la sicurezza che, nel nostro Paese, sono centrali nella difesa della comunità dal crimine. Il continuo sottolineare nei discorsi politici e da parte dei mass media dell'importanza di occuparsi di tutto ciò che potrebbe andare a danneggiare la sicurezza sociale, porta a rinforzare un immaginario collettivo caratterizzato dall'insicurezza, determinando un successivo calo della felicità della popolazione territoriale. Bauman (Bauman, Vecchi, 2004) $)^{51}$, nel descrivere la transizione che una società subisce nel trasformarsi da una sua fase "solida" a quella "liquida", si concentra sugli effetti confusivi e destabilizzanti delle identità personali e collettive, sottolineando come questi possano andare ad intaccare la percezione di "sicurezza ontologica" della società. Le possibili conseguenze di questo senso di insicurezza vanno a gravare su quelle paure che diventano centrali all'attenzione personale e sociale, tra le quali "la paura della criminalità, storicamente e tristemente nota per la sua capacità di condensare e solidificare emozioni personali e sociali presenti allo «stato gassoso» fino a produrre precipitazioni sociali gravide di effetti e conseguenze." ${ }^{52}$

La paura della criminalità va inconsciamente a definire la qualità della vita in quanto condiziona lo stile di vita e la partecipazione attiva al sociale sia del singolo che dell'intera collettività (Santinello, Gonzi, Scacchi, 1998), ${ }^{53}$, influenzando anche lo stato di benessere psicologico essendo essa stessa un fattore di stress ambientale in presenza di vittimizzazione diretta (Oliver et al., 1997)..$^{54}$

In Italia, possibile riscontrare nel concreto quello che Garland $(2001)^{55}$ definiva come crime complex ovvero la "sindrome pericolo criminalità" inquadrabile dalla sussistenza di alcuni elementi quali:

"la normalizzazione sociale di alti indici di criminalità; la presenza di forti emozioni che vanno dall'attrazione alla paura, dal risentimento alla $\mathrm{r}$

abbia; la politicizzazione del problema criminalità anche in termini intensamente emotivi; [...]; la convinzione che la giustizia sia inadeguata e inefficace; [...]; l'istituzionalizzazione del problema del crimine nei mass media, nella cultura popolare $[\ldots]$ ". ${ }^{56}$

All'enfatizzazione del problema che presenta la criminalità come un'emergenza contemporanea in rapida e sempre più pericolosa diffusione, consegue l'amplificazione dell'allarme sociale.

Tornando a parlare di prevenzione, è di dovere fornire una definizione letterale del termine. Dunque, per prevenzione si intende l'adozione di una serie di interventi volti ad anticipare e a evitare che un pericolo o male sociale di varia natura si realizzi nella sua compiutezza; cercando perciò di individuare in che area bisogna agire per raggiungere questo scopo e per impedire, in aggiunta, la riproduzione di condotte socialmente indesiderate attraverso la riabilitazione e la riduzione della recidiva.

È essenziale, in quest'ottica, l'individuazione dei cosiddetti fattori di rischio, ovvero quei segnali precursori di probabili futuri atteggiamenti devianti o criminali dai quali può conseguire l'insorgere di disturbi della condotta. Sono tutti quei fattori che abbiamo già affrontato nella prima parte dell'articolo, elementi chiave nella spiegazione dei processi che regolano lo sviluppo dei comporta- menti, ma che risultano tuttora essere insufficienti per trattare in maniera esaustiva il fenomeno in quanto esistono incongruenze circa ciò che questi fattori sembrano predire e ciò che accade realmente nelle biografie degli individui.

Si testimonia la sussistenza di diversi modelli di approccio in contrapposizione tra loro, dai modelli esplicativi monofattoriali come quelli relativi ai fattori biologici o a quelli socioculturali e relazionali, ad altri modelli proposti in alternativa, ovvero quello additivo e quello interattivo. Questi ultimi ipotizzano un legame tra i diversi fattori di rischio: da una mera unione degli stessi alla quale consegue una maggiore probabilità di sviluppo di un disturbo della condotta, nel primo caso; a una loro interazione che genera conseguenze solo in determinate circostanze per quanto invece riguarda il secondo modello. È così che si è poi ideato il modello mediativo che prende in considerazione $\mathrm{i}$ fattori di rischio nei loro effetti cumulativi e interattivi, ritenendo che $\mathrm{i}$ fattori di rischio distali (biologici, genetici e socioculturali) siano mediati dalle esperienze familiari e dalla qualità delle relazioni con il gruppo dei pari. ${ }^{57}$ Tutti questi modelli coesistono e spesso vengono simultaneamente contemplati per ridurre appunto i fattori di rischio e per potenziare invece i fattori protettivi.

Il comportamento deviante è difficilmente riconducibile ai fattori già elencati, in quanto esito di un processo molto più complesso:

"gli obiettivi che la persona persegue attraverso il comportamento; la percezione della devianza come soluzione possibile a difficoltà considerate eccedenti le proprie risorse, personali e sociali; [...]. Non sono di per sé particolari condizioni sociali, familiari, scolastiche, lavorative, gruppali e particolari caratteristiche individuali a causare linearmente il crimine e gli specifici comportamenti delinquenziali. 亡̀ possibile piuttosto affermare che tutte le condizioni citate divengono attive in senso causale a seconda di come intervengono i cosiddetti sistemi di mediazione autoregolativa (De Leo, Patrizi, 2002, p.88). ${ }^{58,59}$

È possibile affermare che nessuno dei fattori di rischio sia in grado né da solo, né attraverso un effetto moltiplicatore, di dare corso ad un'azione che abbia esito criminale, in quanto tali processi dipendono non solo dai fattori stessi, bensì anche dalla loro interazione reciproca e da come questi vengono regolati dai sistemi di mediazione autoregolativa. ${ }^{60}$

50 Ante delictum (prima che il delitto venga commesso)

51 Bauman Z., Vecchi B. (2004), Identity: Conversations with Benedetto Vecchi, Polity.

52 Patrizi P., Psicologia della devianza e della criminalità. Teorie e modelli di intervento, Carocci editore.

53 Santinello M., Gonzi P., Scacchi L. (1998), Le paure della criminalità. Aspetti psicosociali di comunità, Giuffrè .

54 Oliver J., Huxley P., Bridges K., Mohamad H. (1997), Quality of life and mental health service, Routledge .

55 Garland D. (2001), The culture of control-crime and social control in contemporary society, Oxford University Press; trad. it. (2004) La cultura del controllo, Il Saggiatore.

56 Patrizi P., Psicologia della devianza e della criminalità. Teorie e modelli di intervento, Carocci editore.

57 Patrizi P., Psicologia della devianza e della criminalità. Teorie e modelli di intervento, Carocci editore.

58 De Leo G., Patrizi P. (2002), Psicologia della devianza, Carocci, p. 88.

59 Patrizi P., Psicologia della devianza e della criminalità. Teorie e modelli di intervento, Carocci editore.

60 Il modo in cui le condizioni psicologiche, ambientali, sociali e familiari influenzano la vita dell'individuo e vengono avvertite e sfruttate dalla persona e dai suoi contesti di appartenenza in senso attivo. 
La prevenzione attraverso l'eliminazione e la riduzione dei fattori di rischio ha dunque mostrato diverse complicazioni, dagli elevati costi economici, alla difficoltà di intervenire in maniera tempestiva, al problema di riuscire a comprenderne l'effettiva efficacia. Considerata dunque la progressiva consapevolezza dei limiti concreti del suddetto approccio, si è dimostrata necessaria la ricerca di un nuovo sistema di prevenzione che andasse ad affiancare quello precedente, senza sostituirlo interamente.

È nata così la recente prospettiva promozionale della prevenzione fondata sulla cognizione di un rapporto circolare tra l'agire dell'uomo e il contesto sociale in cui questo interviene, un mondo sia fisico che sociale che ha ripercussioni sulle azioni delle persone, ma che viene a sua volta influenzato e modificato da quelle stesse persone e dai loro comportamenti. Si parla perciò di agentività umana ovvero:

"la capacità dell'individuo di intervenire in senso causale sulla realtà. È questo il significato di una mente "proattiva", di un individuo che agisce sia sul proprio mondo interno che sull'ambiente, trasformando entrambi; lo fa non solo a partire dalle sue precondizioni (la sua storia, le sue caratteristiche di personalità) ma, e soprattutto, in funzione delle sue anticipazioni (De Leo, Patrizi, 2002, p.21)". ${ }^{61}$

Una mente umana che non re-agisce al mondo esterno o interno, ma inter-agisce con essi (Mead, 1934). ${ }^{62}$

In conclusione, per la prevenzione del crimine e della devianza, è fondamentale l'inserimento delle istituzioni all'interno dei quattro livelli di mediazione autoregolativa quali: quello individuale, quello familiare, quello gruppale e infine quello sociale.

Non è quello individuale però il livello oggetto delle maggiori azioni preventive, bensì è bene orientare l'agire verso il sistema familiare, cercando di coinvolgerlo in attività di tipo prosociale e volontaristico. Così come si è dimostrato di estrema importanza l'intervento all'interno del gruppo di pari, soprattutto durante l'adolescenza quale fase della vita in cui è maggiore l'esordio di percorsi devianti, anche se gli adolescenti sono forse i bersagli più complicati in quanto si trovano in una continua e rapida evoluzio- ne, difficile da intercettare e fermare. In questo livello, l'azione di prevenzione deve confluire verso la sottocultura che regola l'ambiente del gruppo e la leadership del gruppo stesso, progettando delle pari opportunità socializzanti e di integrazione in contesti con un minore rischio di criminalità. Bisogna inoltre agire attraverso ricerche di intervento di strada soprattutto in ambienti con alto tasso di immigrazione, dove è necessario instaurare un dialogo aperto di inclusione e di partecipazione della e nella comunità ospitante.

Infine, per quanto riguarda i gruppi formali e informali all'interno delle scuole anche primarie, bisogna promuovere un'aggregazione positiva e soprattutto intercettare quelli che sono segnali e sintomi critici, come per esempio il trasgredire le regole, il non sottostare all'autorità delle figure adulte o atteggiamenti violenti, prevaricatori e prepotenti.

"Prevenire in chiave promozionale la devianza e la criminalità è dunque un complesso e raffinato processo di promozione della qualità della vita di ognuno e di tutti, che interroga tutti noi e la comunità in cui viviamo inclusi coloro che ne trasgrediscono le norme, ma altrettanto possono essere richiamati e re-inclusi nel ripararne gli effetti dannosi." 63

È sul livello della qualità della vita quindi che la prevenzione promozionale ha maggiori risultati, anche in uno scenario nazionale come quello italiano, in cui coesistono povertà di risorse sociale e l'incremento della richiesta di sicurezza; perché è proprio in una comunità rancorosa che la criminalità diventa il principale propulsore del senso di insicurezza, che ne impregna l'aria, rendendo la società stessa la più adatta ad accogliere la criminalità.

Esiste una linea tra il Bene e il Male, i privilegiati amano pensare che questa sia fissa, impermeabile, con loro dalla parte del Bene e il resto della società da quella del male. Ma sappiamo che quella linea è mobile e permeabile. Sappiamo che le persone buone possono essere sedotte oltre quella linea e che, d'altro canto, chi considerato "cattivo" può guarire con un aiuto, con la riabilitazione. Il mondo è, era e sempre sarà colmo di Bene e di Male, lo "yin" e lo "yang" della condizione umana.
Correspondence: Martina Bochicchio.
E-mail: marti.boc.96@gmail.com

Key words: violence; aggression; deviance.

Parole chiave: violenza; aggressività; devianza.

Palabras clave: violencia; agresión; desviación.

Received for publication: 1 June 2020.

Accepted for publication: 2 Juy 2020.

This article is distributed under the terms of the Creative Commons Attribution Noncommercial License (by-nc 4.0) which permits any noncommercial use, distribution, and reproduction in any medium, provided the original author(s) and source are credited.

${ }^{\circ}$ Copyright: the Author(s), 2020

Licensee PAGEPress, Italy

Rivista di Psicopatologia Forense, Medicina Legale, Criminologia $2020 ; 25: 548$

doi:10.4081/psyco. 2020.548
61 De Leo G., Patrizi P. (2002), Psicologia della devianza, Carocci, p. 21.

62 Mead G.H. (1934), Mind, self and society, University of Chicago Press, trad. it (1966), Mente, sé e società.

63 Patrizi P., Psicologia della devianza e della criminalità. Teorie e modelli di intervento, Carocci editore. 